\title{
Effect of Health Systems Strengthening in Influencing Maternal and Neonatal Health Outcomes in Bungoma County, Kenya
}

Beverly Ochieng ( $\nabla$ beverly_ochieng@yahoo.com )

Tropical Institute Of Community Health And Development

Giorgia Lattanzi

hera - right to health and development

Milka Choge

United Kingdom Foreign, Commonwealth and Development Office.

Amarddep Thind

Western University

\section{Research Article}

Keywords: Maternal, Neonatal, Care, Child, Health Systems, Morbidity, Access, Utilization, Quality and Reproductive Health

Posted Date: December 9th, 2020

DOl: https://doi.org/10.21203/rs.3.rs-115746/v1

License: (9) This work is licensed under a Creative Commons Attribution 4.0 International License. Read Full License 


\section{Abstract}

Kenya is estimated to have a population of $\mathbf{4 7 . 6}$ million people. Its maternal and neo natal health indicators have steadily improved over the years. Despite the progress, many significant challenges remain, including persistent inequities between population subgroups, and a health care system that provides variable quality and inconsistent access to care, especially for women and new-borns.

Purpose of this paper was to assess the impact of the maternal and new-born improvement intervention in Bungoma County, with a focus on access and quality of maternal and neonatal care services.

Methodology: The study design was quasi-experimental, using household surveys to assess outcomes.

Findings: Provision of transport vouchers decreased barriers to accessing care, resulting to an increased number of deliveries in health facilities. The health system strengthening intervention possibly had a role in improving potential and actual access to antenatal care and health facility delivery services for mothers as well as positively impacting quality of care provision in the intervention sub-counties. However, actual access for babies (measured by provision of PNC care within 48 hours of delivery) seemed to worsen in the intervention sub-counties.

Conclusion: Our analysis suggests that it is possible to conduct an evaluation of the impact of interventions in messy real-life contexts where data availability is challenging. Key elements of health system strengthening include reducing cost barriers and enhancing the capacity of the health facilities to deliver the high quality care. These can fruitfully be implemented in other regions.

\section{Background}

Kenya is a young and growing country, with an estimated population of 47.6 million in 2019(1). Its health indicators (such as infant mortality rate, under- 5 mortality rate, maternal mortality ratio, etc.) have steadily improved over the years, as evidenced by the 2008 and 2014 demographic survey data.(2) This has been accompanied by its Universal Health Care (UHC) service coverage index being scored at 55, which is relatively high in the context of sub-Saharan Africa.(3) Despite this progress, many significant challenges remain, including persistent inequities between population subgroups, and a health care system that provides variable quality and inconsistent access to care, especially for women and newborns.

The country has responded to these challenges with a series of vigorous reforms in the past decade. The new 2010 constitution introduced 47 semi-autonomous county governments, with substantial devolution of responsibility for health service delivery from the central government to these counties.(4) User-fees for skilled delivery at health facilities were eliminated with the introduction of the Linda Mama program. The Reproductive Maternal New-born Child Adolescent Health (RMNCAH) Investment Framework was launched in 2016to guide smart, scaled up and sustained financing for delivering quality RMNCAH services.(5) More recently, the increasing focus of the Kenyan government on UHC (as one of the four 
pillars of its Big Four Agenda) has provided much needed attention to maternal and child health indicators. However, persistent capacity gaps, especially at the county health system level, stymie further improvement in RMNCAH goals. The newly created county governments often do not have the requisite technical capacity to manage devolved functions effectively, and there is lack of clarity around the roles, responsibilities and structures existing at national and county level and the flow of revenues and resources(6)

Many development partners and donors have supported the country during this period of significant change. One such support is provided by the United Kingdom Foreign, Commonwealth and Development Office (FCDO) funded programme to reduce Maternal and Neonatal Deaths in Kenya (the MNH Programme) which supports increased access to and utilisation of, quality maternal and new-born health services. While the MNH program was national in scope, our analysis focuses on one county (Bungoma).

\section{Bungoma County}

Bungoma County is located in the Western Province and has a population of approximately 1.5 million. The county comprises of ten sub-counties. Its health indicators and health care system are fairly typical of a Kenyan county. However, it is more densely populated, and its per capita GDP is half that of the national average.(1)

In Bungoma, the MNH program support commenced in 2013 and had two broad components supporting health systems strengthening and demand creation, with a focus on maternal and new-born health services. This came to be known as the- Maternal and New-born Improvement, Health Systems Strengthening (MANI-HSS) programme and was implemented in 6 of the ten sub-counties. A range of activities were implemented at the county and sub-county level, in close partnership with the county health authorities and the county-led donor coordination forum. Activities in the six counties included, supporting health facilities by providing EmONC training to staff and establishment of six technical working groups (TWGs) to address human resources for health, health care financing, quality of service delivery, monitoring and evaluation, community health services, and health products and technology management respectively. (7)

At sub-county level, activities included support for annual work plans and budgets, application of the organisational capacity assessment tool, orientation and training of Hospital Management Boards, support for health facility planning and budgeting, orientation and training of Health Facility Management Committees, and provision of travel vouchers for pregnant women to deliver at healthcare facilities.

The purpose of this paper is to assess the impact of the MANI HSS intervention in Bungoma County, with a focus on access and quality of maternal and neonatal care services.

\section{Methods}

\section{Study design}


Figure 1 depicts the quasi-experimental study design, which was necessitated due to data availability (and lack thereof). The classic pre-post with control group design requires baseline and end line data for both intervention and control groups.(8)The MANI programme was implemented in six sub-counties (Kabuchai, Kanduyi, Sirisia, Tongaren, Webuye East and Webuye West); the remaining four sub-counties (Bumula, Mt. Elgon, Kimilili and Cheptais) were treated as the controls. Data was available for the end line (2018) in both intervention and control sub-counties; but baseline data (2015) was only available for the intervention counties. (See Fig. 1)

As baseline data for the control group was not available, we were not able to utilize the pre-post with control group design and analysis. Instead, we conducted two separate analyses:

(a) before/after analysis in the intervention sub-counties, i.e. comparing the end line survey results in 2018 with the results of the baseline survey conducted in 2015 in the same sub-counties. This was to find out if, and to what extent, the MNH indicators in intervention sub counties areas had changed; and (b) an end line analysis comparing control and intervention sub-counties.

While this design does not address all threats to validity, a statistically significant change in a variable observed in both analyses (i.e. baseline vs. end line and intervention vs. control at end line) was taken as an indicator of change possibly attributable to the intervention.

\section{Study population and data collection}

For the 2018 end line surveys, 448 women aged 18-45 years were interviewed in each of the intervention and control groups. For the 2015 baseline, we had data on 478 women in the intervention group; as previously described, no data were available for the control group baseline.

In order to generate the sample for the end line household survey, eight health facilities in the intervention sub-counties were matched with eight health facilities in the control sub-counties based on ownership, level, type and service provision characteristics. The service areas of each health facility were mapped using census data, and stratified cluster sampling was employed to identify households for interview. Inclusion criteria were having delivered a live infant, had a still birth or a third trimester spontaneous abortion within 12 months of the survey.

The baseline survey questionnaire examined variables that assessed access utilization and quality of $\mathrm{MNH}$ services in the intervention areas of the study. The questionnaires for both baseline and end line surveys were developed by adapting the Demographic and Health Survey questionnaires to the indicators relevant to the $\mathrm{MNH}$ program and were closely aligned. In addition to demographics, questions focused on antenatal care attendance, delivery by skilled providers, access to care and perception of service quality and level of satisfaction with MNH services.

\section{Variables}


Variables collected during the surveys were grouped into five domains - a) mother's potential access; $b$ ) mother's actual access; c) baby's actual access; d) quality of care received by the mother; and e) quality of care received by the baby.

a. Potential access (mother): Costs are a recognized barrier to health care access, and interventions at the national level (elimination of user fees under the Linda Mama scheme) and local levels (provision of transport vouchers as part of the intervention) aimed to decrease, if not eliminate, this barrier. We assessed expenditures for ANC services, transport to health facility, and delivery services as binary variables (zero, some expenditure).

b. Actual access (mother): The goal of the interventions was to increase utilization of health services or improve the actual (i.e. realized) access. Maternal access to services was captured by asking women whether they attended any ANC clinic during their last pregnancy, whether they had at least 4 ANC contacts during the last pregnancy, whether an ANC contact occurred in the first trimester, whether she delivered in a health facility and whether she received post-natal care (PNC) services within 48 hours of the delivery. We dichotomized all variables (no/yes).

c. Actual access (baby): This was evaluated with a binary variable (no/yes) whether post-natal care was provided within 48 hours of birth.

d. Quality of care (mother): Mothers were asked if they delivered via C-section, and whether they were satisfied with the ANC care received. These variables were binary (no/yes). Quality of ANC was assessed by asking women about having received 14clinical interventions or provider advice as part of the service; ranging from measuring blood pressure to advice on nutrition. Thirteen or more positive answers were coded as high quality, 7 to 12 were coded as medium and six of less were coded as poor quality. Quality of postnatal care (PNC) to the mother was assessed by whether they had received nine clinical interventions or provider advice as part of the first PNC examination. These ranged from measuring blood pressure to advice about breastfeeding. Depending on the number of positive responses, this was coded as high (7 or more), medium (5-6) or low (4 or less).

e. Quality of care (baby): was captured with two variables. A dichotomous variable (no/yes) captured whether breastfeeding was initiated in the first hour. Quality of PNC provided to the child was assessed by asking mothers whether the child received six clinical interventions or provider advice as part of the first PNC examination, ranging from a physical examination to information about signs and symptoms that suggest that the baby is unwell. Six positive answers were coded as high quality, four and five as medium quality and three or less as low quality.

As all variables were categorical, we used Pearson's Chi-Square or in cases of small numbers, Fisher's Exact tests.

All study participants provided written assent to participate. The study protocols were reviewed and approved by relevant institutional ethics review boards in Kenya (Nairobi University) and Kenyatta National Hospital Ethics Review Boards. The national research clearance certificate and permit was obtained from National Commission for Science, Technology and Innovation (NACOSTI). All methods 
were performed in accordance with relevant guidelines and regulations of Kenya that protected the research subjects. The committees had the right to monitor the research while ongoing, after the end of the study the researchers submitted a final report to the two committees containing a summary of the study findings and conclusions.

\section{Results}

Baseline (intervention only): A total of 478 women (age 15-49 years) were interviewed in 2015 baseline survey. Their mean age was 25.9 years, and a majority had completed primary education $(41 \%)$ or had no education/not completed primary education (36\%). The mean wealth index score (on a scale of 0-2.1) was 0.85 (SD 0.1).

End line (intervention and control): A total of 896 women (age 15-49 years) were interviewed, 448 from MANI and 448 from control areas. The mean age of respondents was 26.4 years in the intervention and 26.2 years in the control area. More than a third (35\%) of respondents had completed primary education in the intervention area; the corresponding number was $42 \%$ in the control area. The mean wealth index score (on a scale of 0-2.1) was 0.833 (SD:0.230) in intervention and 0.901 (SD:0.244) in control area.

\section{Before/after analyses}

Table 1 shows the results of the baseline vs. end line analysis for the intervention group. Among the potential access variables, statistically significant change was noted for transport expenditure, with a greater proportion of women at end line reporting zero expenditure. Statistically significant changes were noted for ANC services, with a greater proportion of women at end line reporting at least once ANC contact, at least 4 ANC contacts, and first ANC contact in the first trimester, respectively, compared to the baseline group. Women in the end line group were also more likely to deliver at a health facility. However, children in the end line group were less likely to get postnatal care within 48 hours as compared to the baseline group. (See Table 1)

Statistically significant changes were also noted for quality variables. Women in the end line group were more likely to report receipt of quality care, and more like to report satisfaction with ANC received, compared to baseline. A greater proportion of the end line respondents reported initiation of breastfeeding in the first hour, and improved quality of postnatal care, as compared to the baseline group.

No statistically significant differences were noted for personal expenditures for ANC services, receipt of postnatal care to mother within 48 hours, the $\mathrm{C}$-section rate and the quality of post-natal care provided to the child between the two groups. (See Table 1)

\section{Intervention vs. control analyses}

Table 2 shows the results of the end line survey analysis for the intervention and control group. Among the potential access variables, statistically significant change was noted for personal expenditure for ANC service and expenditure for transport to the health facility for delivery. A greater proportion of women in 
intervention areas reported no expenditure for ANC services and transport to the health facility to deliver respectively, compared to control areas. (See Table 2)

Statistically significant changes were noted for health facility delivery, with a greater proportion of women in the intervention areas reported having delivered in a health facility than in the control areas. However, women and children in the intervention areas were less likely to receive postnatal care within 48 hours as compared to the control sites; this finding was statistically significant. Statistically significant changes were also noted for quality of care variables. Women in the intervention areas were more likely to report receipt of high quality ANC care and receipt of better quality maternal postnatal care, compared to women in to the control areas.

However, better quality child service provision was reported in control areas compared to intervention areas. A greater proportion of mothers in control areas reported a postnatal check-up of their infants than in project areas. A larger proportion of mothers in control areas reported high quality PNC child services compared to intervention areas. These differences were statistically significant.

No statistically significant differences were noted between MANI intervention and control areas for personal expenditure for delivery, ANC contact in the first trimester of pregnancy, at least four ANC, satisfaction with the antenatal care received, C-section rate, initiation of breastfeeding within the first hour, at least three postnatal contacts for the child within the first two months and satisfaction with the maternity care received (See Table 2)

\section{Discussion}

Table 3 presents the results in a summative manner. The ' + ' indicates that the relationship is significant and positive (in the expected direction); the '- 'indicates a statistically significant relationship but in the opposite direction. A blank cell denotes that the relationship was not statistically significant.

A variable that is statistically significant in both analyses is taken as an indicator of change possibly attributable to the intervention. Based on this decision rule, the intervention possibly had a role in improving potential and actual access to antenatal care and health facility delivery services for mothers as well as positively impacting quality of care provision.(See Table 2)

It was no surprise that the intervention had an impact on transport expenditure, as focused provision of transport vouchers was a key element of the intervention. Beneficiaries of the voucher scheme were poorer women identified in intervention communities, who were reached by a community health worker with information on birth preparedness and received a transport voucher in their 3rd trimester of pregnancy. The voucher scheme covered return transportation to the health facilities for the mother and one companion, which was provided by boda-boda (motorcycle taxi) drivers affiliated to the programme, thus reducing financial barriers to care. In addition, by making a reliable and fast means of transportation available for free to women, the intervention also addressed the second delay in the three delays model, i.e. delay in accessing care, which can further contribute to improving maternal and neonatal health.(9) 
In terms of realized access, the intervention seemed to have facilitated deliveries in health facilities. Given that the intervention activities included demand creating activities such as

re-orienting traditional birth attendants into birth companions, health education sessions aimed at changing views on skilled birth attendance, and community scorecard dialogues aimed at promoting social accountability, our finding is eminently plausible. However, it is wise to keep in mind changes in the contextual environment that could have also played a role. A major environmental factor was the abolition of user fees for maternity care, enacted by the government in 2016. Literature suggests that this led to the increase of facility based deliveries from $44 \%$ (in 2012/13) to $62 \%$ (in 2016).(10). Our study design did not allow us to disentangle these factors. Taken together, the voucher and the user fee elimination are key to enhancing social justice, especially when placing a priority on the most vulnerable, and makes eminent economic sense $(11,12)$

The intervention package also seemed to have a positive impact on quality of care, as measured by quality of ANC and PNC received by mothers; however, our results indicate that it did not have the same impact on actual access for the baby, where we found a negative impact on whether postnatal care was provided to the baby within 48 hours of delivery. This could be plausible for a few reasons.

First, it is possible that the MANI intervention with its goal of health systems strengthening (e.g. providing EmONC training and refreshers, TWG focusing on service delivery, application of organizational capacity assessment tools, etc.) could have had a greater focus and emphasis (especially in implementation) on the mothers compared to their babies, thus resulting a in a differential impact. .

Second, literature indicates that while women are generally able to report accurately on aspects of postnatal care received, indicators related to new-born care received by the child are subject to greater recall bias than those related to postnatal care received by the mother. (13) A recent review of populationbased survey data on maternal and new-born care in 20 Sub-Saharan Africa countries showed that approximately two-thirds (65\%) of women and their babies had received some form of postnatal care, only $3 \%$ reported receiving all seven interventions included in the analysis(14)

Third, it is important to keep in mind the context of Bungoma County, where several projects of the MNH Programme's County Innovation Challenge Fund (CICF) were implemented at the same time as the MANI HSS project. Most notably, two projects focusing on neonatal care by Save the Children International (SCl) and by Mount Kenya University (MKU) were active in both intervention and control sub-counties. In addition, the Liverpool School of Tropical Medicine (LSTM) was implementing its training programme 'Making it Happen' $(\mathrm{MiH})$ in the region. In this dynamic environment, marginal end-user changes that could be attributed to the MANI HSS project would be difficult to detect, especially with our design.

\section{Limitations}

Our study has some clear limitations. Our design is handicapped by the lack of baseline data for the control group precluding the creation of a true counterfactual. This does not allow us to control for 
specific threats to internal validity such as maturation. We are also limited in terms of data and its availability. Baseline data for the intervention sub-counties was limited to an existing survey, and to ensure comparability, we were restricted to the same questions (and responses) used in the existing survey. In addition, the policy environment and geographic context in which the interventions were implemented was not static, making clear attribution challenging due to a multiplicity of programmes.

\section{Conclusion}

Despite these limitations, our analysis suggests that it is possible to conduct an evaluation of the impact of interventions in messy real-life contexts where data availability is challenging. Our results suggest that the MANI HSS intervention could plausibly have had apositive impact on improving access to care and providing better quality of care to pregnant mothers in Bungoma County. Key elements include reducing cost barriers to generate demand and enhancing the capacity of the health facilities to deliver the high quality care. These can fruitfully be implemented in other regions.

\section{Abbreviations}

ANC- Ante Natal Care

CICF- County Innovation Challenge Fund

DFID - Department for International Development

EmONC - Emergency Obstetric and Neonatal Care

GDP - Gross Domestic Production

HSS - Health Systems Strengthening

LSTM - Liverpool School of Tropical Medicine

$\mathrm{MiH}$ - Making it Happen

MNH- Maternal Neonatal Health

MANI - Maternal and New-born Improvement

MKU - Mount Kenya University

NACOSTI - National Commission for Science, Technology and Innovation

PNC - Post-Natal Care

RMNCAH - Reproductive Maternal New-born Child Adolescent Health 
$\mathrm{SCl}$ - Save the Children International

TWGs -Technical Working Groups

UHC- Universal Health Care

\section{Declarations}

The publication costs associated with this article are funded by the United Kingdom Foreign, Commonwealth and Development Office.

\section{Ethics approval and consent to participate}

All participants provided written consent to participate in the study.

\section{Consent for publication}

All authors read and approved the final manuscript.

\section{Availability of data and materials}

The datasets used and/or analyzed during the current study are available from the corresponding author on request.

\section{Competing interests}

The authors declare no conflict of interest

\section{Funding}

This study was funded by the United Kingdom Foreign, Commonwealth and Development Office.

\section{Project Number}

40123716

\section{Authors Contribution}

BMO: Drafted the methods, results and discussion sections. She also synthesized the contributions of the other authors into the first draft of the manuscript. She further engaged actively in the revision of the manuscript in response to critiques from co-authors and took the lead in editing the manuscript based on internal peer reviewers' comments.

GL: Drafted the background and contributed in drafting the discussion section. She reviewed and contributed to the editing of the final manuscript draft and made suggestions and inputs towards the final draft. 
MC: Contributed in drafting the background section

AT: Supervised the writing of the paper and the writing team, spearheaded data analysis and synthesized the contributions from other authors into the manuscript.

\section{Acknowledgements}

This work was funded and supported by the United Kingdom Foreign, Commonwealth and Development Office.

The evaluation research work was carried out by HERA.

We thank Dr. Leo Deville, Dr. Josef Decosas, and Prof Dan Kaseje for reviewing and commenting on earlier drafts of this paper.

\section{References}

1. Kenya National Bureau of Statistics. Gross County Product 2019. 2019.

2. Kenya. Ministry of Health., Kenya National Bureau of Statistics., National AIDS Control Council (Kenya), Kenya Medical Research Institute., National Council for Population and Development (Kenya: 2012), DHS Program. Kenya demographic and health survey, 2014. Nairobi, Kenya: Kenya National Bureau of Statistics; 2015. xxvi, 575 pages $\mathrm{p}$.

3. WHO. Global Health Observatory data repository. Index of service coverage, data by country WHO; 2018 [Available from: https://apps.who.int/gho/data/view.main.INDEXOFESSENTIALSERVICECOVERAGEv.

4. Kenya LO. The Constitution of Kenya: 2010: Chief Registrar of the Judiciary; 2013.

5. Ministry of Health GoK. Kenya Reproductive, Maternal, Newborn, Child and Adolescent Health (RMNCAH) Investment Framework. 2016 January 31, 2016.

6. Tsofa B, Molyneux S, Gilson L, Goodman C. How does decentralisation affect health sector planning and financial management? A case study of early effects of devolution in Kilifi County, Kenya. International journal for equity in health. 2017;16(1):151.

7. TICH, HERA. MNH Programme. Summative Evaluation Final Report. 2020.

8. Campbell DT, Stanley JC. Experimental and quasi-experimental designs for research: Ravenio Books; 2015.

9. Thaddeus S, Maine D. Too far to walk: maternal mortality in context. Social science \& medicine. 1994;38(8):1091-110.

10. Masaba BB, Mmusi-Phetoe RM. Neonatal Survival in Sub-Sahara: A Review of Kenya and South Africa. Journal of multidisciplinary healthcare. 2020;13:709.

11. Frenk J, De Ferranti D. Universal health coverage: good health, good economics. The Lancet. 2012;380(9845):862-4. 
12. Powers M, Faden RR, Faden RR. Social justice: the moral foundations of public health and health policy: Oxford University Press, USA; 2006.

13. McCarthy KJ, Blanc AK, Warren CE, Mdawida B. Women's recall of maternal and newborn interventions received in the postnatal period: a validity study in Kenya and Swaziland. Journal of global health. 2018;8(1).

14. Carvajal-Aguirre L, Amouzou A, Mehra V, Ziqi M, Zaka N, Newby H. Gap between contact and content in maternal and newborn care: An analysis of data from 20 countries in sub-Saharan Africa. Journal of global health. 2017;7(2).

\section{Tables}

Table 1: Baseline Vs. End line analyses 


\begin{tabular}{|c|c|c|c|c|}
\hline \multirow[t]{2}{*}{ Indicator } & \multicolumn{2}{|c|}{ Baseline } & \multicolumn{2}{|c|}{ End line } \\
\hline & $\mathrm{N}$ & $\%$ & $\mathrm{~N}$ & $\%$ \\
\hline a) Potential access (mother) & & & & \\
\hline \multicolumn{5}{|l|}{ Expenditure for ANC services } \\
\hline Zero & 181 & 78 & 287 & 74 \\
\hline Some expenditure & 52 & 22 & 102 & 26 \\
\hline \multicolumn{5}{|c|}{ Expenditure for transport to the health facility fordelivery** } \\
\hline Zero & 0 & 0 & 39 & 16 \\
\hline Some expenditure & 261 & 100 & 198 & 84 \\
\hline \multicolumn{5}{|l|}{ Expenditure fordelivery $\star \star$} \\
\hline Zero & 97 & 59 & 314 & 84 \\
\hline Some expenditure & 67 & 40 & 58 & 16 \\
\hline b) Actual access (mother) & & & & \\
\hline \multicolumn{5}{|c|}{ At least one ANC contact with a formalprovider** } \\
\hline No & 32 & 7 & 3 & 1 \\
\hline Yes & 446 & 93 & 389 & 99 \\
\hline \multicolumn{5}{|c|}{ At least four ANC contacts with a formalprovider** } \\
\hline No & 221 & 46 & 102 & 26 \\
\hline Yes & 257 & 54 & 290 & 74 \\
\hline \multicolumn{5}{|c|}{ ANC contact in the first trimester ofpregnancy** } \\
\hline No & 376 & 79 & 274 & 70 \\
\hline Yes & 100 & 21 & 118 & 30 \\
\hline
\end{tabular}




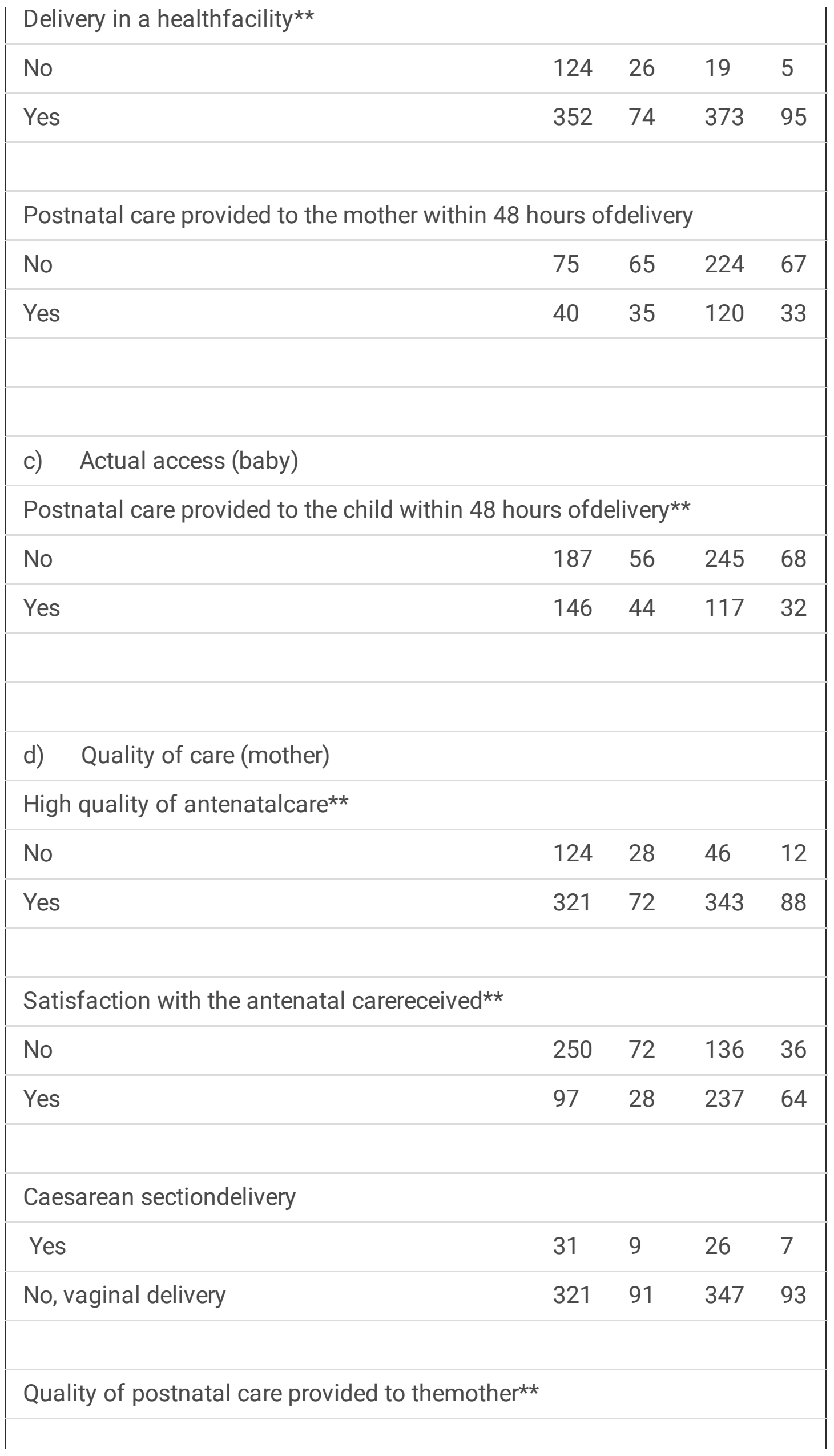

Page $14 / 20$ 


\begin{tabular}{|lllll|}
\hline Low & 86 & 57 & 48 & 38 \\
\hline High & 31 & 21 & 66 & 53 \\
& 33 & 22 & 11 & 9 \\
\hline e) Quality of care (baby) & & & & \\
\hline Quality of postnatal care provided to thechild & & & & \\
\hline Low & 9 & 36 & 31 & 26 \\
\hline Medium & 8 & 32 & 37 & 31 \\
\hline High & 8 & 32 & 51 & 43 \\
\hline Initiation of breastfeeding within the firsthour** & & & & \\
\hline No & 153 & 48 & 113 & 33 \\
\hline Yes & 168 & 52 & 228 & 67 \\
\hline
\end{tabular}

$\star * p<0.05$

Table 2: Intervention Vs. Control group results 


\begin{tabular}{|c|c|c|c|c|}
\hline \multirow[t]{2}{*}{ Indicator } & \multicolumn{2}{|c|}{ Intervention } & \multicolumn{2}{|c|}{ Control } \\
\hline & $\mathrm{N}$ & $\%$ & $\mathrm{~N}$ & $\%$ \\
\hline \multicolumn{5}{|l|}{ a) Potential access (mother) } \\
\hline \multicolumn{5}{|c|}{ Expenditure for ANC services** } \\
\hline Zero & 322 & 73 & 270 & 61 \\
\hline Some expenditure & 121 & 27 & 172 & 39 \\
\hline \multicolumn{5}{|c|}{ Expenditure for transport to the health facility fordelivery** } \\
\hline Zero & 42 & 15 & 8 & 3 \\
\hline Some expenditure & 237 & 85 & 229 & 97 \\
\hline \multicolumn{5}{|l|}{ Expenditure fordelivery } \\
\hline Zero & 361 & 86 & 320 & 82 \\
\hline Some expenditure & 61 & 14 & 70 & 18 \\
\hline \multicolumn{5}{|l|}{ b) Actual access (mother) } \\
\hline \multicolumn{5}{|c|}{ At least one ANC contact with a formalprovider } \\
\hline No & 4 & 1 & 7 & 2 \\
\hline Yes & 444 & 99 & 441 & 98 \\
\hline \multicolumn{5}{|c|}{ At least four ANC contacts with a formalprovider } \\
\hline No & 114 & 25 & 134 & 30 \\
\hline Yes & 334 & 75 & 314 & 70 \\
\hline \multicolumn{5}{|c|}{ ANC contact in the first trimester ofpregnancy } \\
\hline No & 316 & 71 & 329 & 73 \\
\hline Yes & 132 & 29 & 119 & 27 \\
\hline
\end{tabular}




\begin{tabular}{|c|c|c|c|c|}
\hline \multicolumn{5}{|c|}{ Delivery in a healthfacility $\star \star$} \\
\hline No & 25 & 6 & 58 & 13 \\
\hline Yes & 423 & 94 & 390 & 87 \\
\hline \multicolumn{5}{|c|}{ Postnatal care provided to the mother within 48 hours ofdelivery $\star *$} \\
\hline No & 258 & 61 & 172 & 44 \\
\hline Yes & 165 & 39 & 218 & 56 \\
\hline \multicolumn{5}{|c|}{ c)Actual access (baby) } \\
\hline \multicolumn{5}{|c|}{ 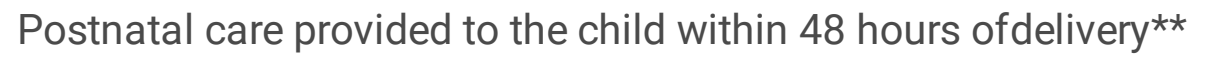 } \\
\hline No & 254 & 88 & 130 & 73 \\
\hline Yes & 36 & 12 & 47 & 27 \\
\hline \multicolumn{5}{|c|}{ d) Quality of care (mother) } \\
\hline \multicolumn{5}{|c|}{ Quality of antenatalcare ${ }^{\star \star}$} \\
\hline No & 205 & 46 & 243 & 55 \\
\hline Yes & 239 & 54 & 198 & 45 \\
\hline \multicolumn{5}{|c|}{ Satisfaction with the antenatal carereceived } \\
\hline No & 47 & 11 & 33 & 7 \\
\hline Yes & 397 & 89 & 408 & 93 \\
\hline \multicolumn{5}{|c|}{ Caesarean sectionrate } \\
\hline Yes & 29 & 7 & 29 & 7 \\
\hline No & 394 & 93 & 363 & 93 \\
\hline
\end{tabular}




\begin{tabular}{|lcccc|}
\hline Quality of postnatal care provided to themother** & & \\
\hline Medium & 70 & 42 & 120 & 55 \\
\hline High & 83 & 50 & 84 & 39 \\
\hline & 12 & 7 & 14 & 6 \\
\hline & & & \\
\hline e) Quality of care (baby) & & & & \\
\hline Quality of postnatal care provided to thechild** & & \\
\hline Low & 45 & 27 & 27 & 11 \\
\hline Medium & 56 & 34 & 118 & 47 \\
\hline High & 63 & 38 & 105 & 42 \\
\hline & & & & \\
\hline Initiation of breastfeeding within the firsthour & & \\
\hline No & 119 & 31 & 101 & 29 \\
\hline Yes & 266 & 69 & 253 & 71 \\
\hline
\end{tabular}

$* * p<0.05$

Table 3: Overall summative results 
$\mathrm{MNH}$ Indicators

a) Potential access (mother)

Expenditure for ANC services

Expenditure for transport to the health facility fordelivery

Expenditure fordelivery

b) Actual access (mother)

At least one ANC contact with a formalprovider

At least four ANC contacts with a formalprovider

First ANC contact in the first trimester ofpregnancy

Delivery in a healthfacility

Postnatal care provided to the mother within 48 hours ofdelivery

c)Actual access (baby)

Postnatal care provided to the child within 48 hours ofdelivery

d) Quality of care (mother)

Quality of antenatalcare

Satisfaction with the antenatal carereceived

Caesarean sectiondelivery

Quality of postnatal care provided to themother

f) Quality of care (baby)

Quality of postnatal care provided to thechild

Initiation of breastfeeding within the first hour,
Baseline vs. End line
Intervention vs. Control

2
+
+


Baseline

Intervention

Control
End line

$\mathrm{O}_{3}$

Figure 1

Study design 